\title{
REDUÇÃO DO CARBONO ORGÂNICO TOTAL (COT) A PARTIR DA OXIDAÇÃO DA LACTOSE EM ÁGUA SUPERCRÍTICA
}

\author{
L. FERREIRA-PINTO ${ }^{1}$, J.C. LIMA ${ }^{1}$, A. F. ZANETTE ${ }^{1}$, P. F. COLDEBELLA ${ }^{1}$, R. \\ BERGAMASCO $^{1}$; D. MANTOVANI ${ }^{1}$; L. CARDOZO-FILHO ${ }^{1}$. \\ ${ }^{1}$ Universidade Estadual de Maringá, Departamento de Engenharia Química \\ E-mail para contato: leandrofpinto@gmail.com
}

\begin{abstract}
RESUMO - Este trabalho reporta dados experimentais da redução de COT (carbono orgânico total) através oxidação da lactose em água supercrítica com ou sem a presença de sais alcalinos $\left(\mathrm{NaOH}, \mathrm{KOH}\right.$ e $\left.\mathrm{Na}_{2} \mathrm{CO}_{3}\right)$ ou oxidantes $\left(\mathrm{H}_{2} \mathrm{O}_{2}\right.$ e t-BHP) em um reator de fluxo continuo, construído em Inconel 625. As reações de oxidação, livres de sais alcalinos ou oxidantes, foram realizadas em uma faixa de temperatura de $550-700{ }^{\circ} \mathrm{C}$, pressão de $22,5 \mathrm{MPa}$, concentrações de lactose na faixa de $0,5 \mathrm{wt} \%$ 2,5 wt\%, fluxos de alimentação de 5 g. min $^{-1}$ e 7 g.min-1 e tempo de reação de 30 minutos. A redução de COT variou entre 50 - 99\%. A melhor condição de degradação do COT $\left(700^{\circ} \mathrm{C}\right.$ e $\left.0,5 \mathrm{wt} \%\right)$ apresentou redução de COT de $\sim 95 \%$. Contudo, a pior condição $\left(550{ }^{\circ} \mathrm{C}\right.$ e $\left.2,5 \mathrm{wt} \%\right)$ apresentou redução de COT de $\sim 50 \%$. Nessa condição operacional a utilização de catalisadores ou oxidantes elevou a redução do COT para $\approx 99 \%$. Os resultados obtidos neste trabalho podem servir como base no estudo da degradação de efluentes lácteos provindos da indústria de laticínios a partir da oxidação em água supercrítica.
\end{abstract}

\section{INTRODUÇÃO}

A oxidação/gaseificação em água supercrítica é um processo que ocorre a altas temperaturas e pressões na destruição eficiente de uma diversidade de resíduos orgânicos aquosos (Savage, 1999). Esse processo ocorre em condições acima do ponto crítico da água $\left(\mathrm{T}_{\mathrm{c}} \geq 374{ }^{\circ} \mathrm{C}\right.$, $P_{c} \geq 22,1 \mathrm{MPa}$ ) (Brunner, 2009). Sob estas condições, a água supercrítica apresenta propriedades físico-químicas únicas, que a tornam um meio eficaz para a oxidação de compostos orgânicos (García Jarana et al., 2008). Assim, as limitações de transporte de massa são minimizadas, levando a altas taxas de reação, com conversões próximas a $100 \%$ para tempos de residência curtos e reatores de pequeno porte (Abelleira et al., 2013; Veriansyah and Kim, 2007; Xu et al., 2012). Além disso, como as reações de oxidação são exotérmicas e endotérmicas, até mesmo a produção de calor pode ser alcançada neste processo (Abelleira et al., 2013; Bermejo et al., 2004; Cocero et al., 2002; Hellström et al., 2008; Jimenez-Espadafor et al., 2011; Silva et al., 2004; Smith and Wang, 2003).

Diante destas características, e por não necessitar que os efluentes passem por tratamentos prévios sofisticados (Abelleira et al., 2013), a aplicação desta tecnologia torna-se um grande 


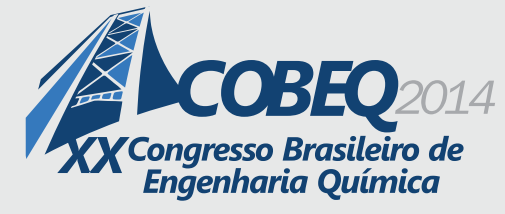

19 a 22 de outubro de 2014

Florianópolis/SC

escape às tecnologias convencionais de tratamento de efluentes industriais ou águas residuais municipais. No caso deste estudo, será abordado o tratamento de efluentes de laticínios, pois, a indústria de laticínios se destaca como um potencial de geração de efluentes. E conectados aos mais diversos tipos de produtos gerados (leite, bebidas lácteas, queijo, doces, sorvetes, cremes, entre outros), torna-se um grande problema, tanto ambiental quanto econômico (Brião e Tavares, 2012, 2007; Glithero et al., 2013).

Os efluentes gerados por essas indústrias é um significante poluidor, por apresentar, em geral, grande concentração de matéria orgânica (carboidratos, proteínas e gorduras) e inorgânica e seu lançamento em corpos hídricos pode diminuir a concentração de oxigênio dissolvido (Demirel et al., 2005; Saraiva et al., 2009).

Produtos como o queijo, dependendo do processo, geram até 10 litros de efluente (soro de leite) para $1 \mathrm{~kg}$ de queijo processado (Brião e Tavares, 2012; Sögüt et al., 2011). Além disso, a demanda de água necessária para geração dos produtos lácteos é um dos grandes problemas enfrentados pelas indústrias de laticínios (Saraiva et al., 2009). Dependendo do tipo de instalação, do sistema de limpeza e seu gerenciamento, a quantidade de água consumida pode ultrapassar consideravelmente o volume de leite processado. Estima-se que o consumo médio de água pode chegar até 10 litros/kg de leite recebido na indústria (Brião e Tavares, 2012; Vourch et al., 2005). No entanto, cerca de $80 \%$ (Brião e Tavares, 2012, 2007) deste consumo estão relacionados à sanitização de laticínios. Reporta-se ainda que neste processo podem ser perdidos até $3 \%$ do volume de leite processado (Vourch et al., 2008).

Além do tratamento deste efluente, uma alternativa de aproveitamento energética é a geração de hidrogênio a partir da gaseificação supercrítica. Os efluentes provindos das indústrias do leite apresentam aproximadamente $90 \%$ de água e grandes quantidades de açucares, sendo um ótimo efluente para oxidação/gaseificação em condições supercríticas.

Desta forma, com a finalidade de agregar informações ao processo de oxidação (supercritical water oxidation - ScWO) de efluentes da indústria de laticínios, este trabalho teve como objetivo oxidar a lactose (composto modelo) em água supercrítica, utilizando sais alcalinos ( $\mathrm{NaOH}, \mathrm{KOH}$ e $\mathrm{Na}_{2} \mathrm{CO}_{3}$ ) e oxidantes (peróxido de hidrogênio e tert-butyl hydroperoxide), tendo como foco a sua degradação.

As reações de oxidação, livres de sais alcalinos ou oxidantes, foram realizadas em uma faixa de temperatura de $550-700{ }^{\circ} \mathrm{C}$, pressão de $22,5 \mathrm{MPa}$, concentrações de lactose na faixa de 0.5 wt $\%$ - 2.5 wt\%, fluxos de alimentação de 5 g. $\min ^{-1}$ e 7 g.min ${ }^{-1}$ e tempo de reação de 30 minutos. A redução de COT variou entre $50-99 \%$. A melhor condição de degradação do COT $\left(700^{\circ} \mathrm{C}\right.$ e $\left.0.5 \mathrm{wt} \%\right)$ apresentou redução de COT de $\sim 95 \%$. Contudo, a pior condição $\left(550{ }^{\circ} \mathrm{C}\right.$ e 2.5 wt $\%$ ) apresentou redução de COT de $\sim 50 \%$. Nessa condição operacional a utilização de catalisadores ou oxidantes elevou a redução do COT para $\approx 99 \%$.

\section{EXPERIMENTAL}




\subsection{Materiais}

A lactose monoidratada ( $>98.0 \mathrm{wt} \%$ ) utilizada neste estudo foi obtida através da Anidrol (Brazil). $\mathrm{O} \mathrm{KOH}, \mathrm{NaOH}$ e $\mathrm{Na}_{2} \mathrm{CO}_{3}$ foram obtidos através da Synth (Brazil). O peróxido de hidrogênio $\left(\mathrm{H}_{2} \mathrm{O}_{2}\right) \sim 30 \mathrm{wt} \%$ e tert-butil hidroperoxido (t-BHP) $\sim 70 \mathrm{wt} \%$ foram obtidos a partir da Synth (Brazil).

\subsection{Aparato e procedimento experimental}

O esquema do aparato experimental utilizado para produção de hidrogênio é apresentado na Figura 1, sendo composto por um reator de fluxo contínuo construído em Inconel 625, préaquecedor, condensador e tubulações, construídos em aço inoxidável 316.

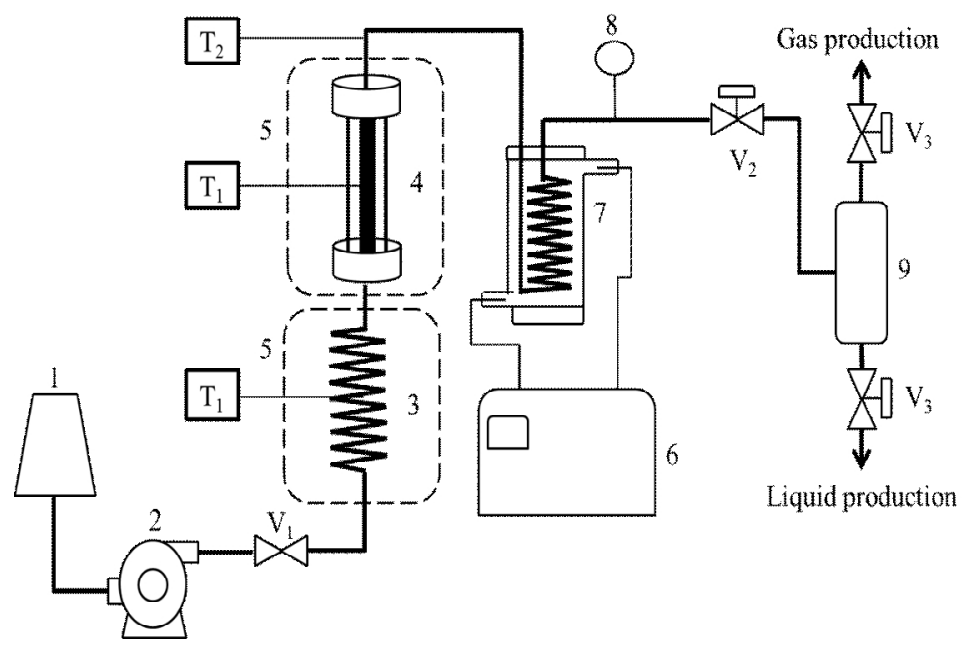

Figura 1 - Esquema do aparato de oxidação em água supercrítica. 1- Reservatório de alimentação;

2- Bomba de alta pressão; 3- Pré-aquecedor; 4- Reator; 5- Forno bipartido; 6- Banho termostatizado; 7- Condensador; 8- Manômetro; 9- Separador de fases; T1- Controlador de temperatura; T2- Indicador de temperatura; V1- Válvula de via-única; V2- Válvula Backpressure; e V3- Válvula.

O procedimento experimental inicia-se com o preparo da solução de lactose, onde quantidades de lactose são pesadas em uma balança de alta escala de precisão (Ohaus Analytical Standard, precisão: $0,0001 \mathrm{~g}$ ). Após, a mistura reacional contida em um reservatório de vidro (1) é succionada por meio de uma bomba de alta pressão, tipo HPLC (2). A mistura é transportada através de um tubo de aço inoxidável $316(L=1,5 \mathrm{~m}, \mathrm{I} . \mathrm{D} .=3,175 \mathrm{~mm})$ até a válvula $\mathrm{V}_{1}$ (válvula de via única) e em seguida distribuída até o pré-aquecedor (3), $(L=6 \mathrm{~m}$, I.D. 3,175 mm). A mistura reacional, devidamente aquecida $\left(50^{\circ} \mathrm{C}\right)$, entra no reator $(4)(L=0.2 \mathrm{~m}$, I.D. $=13 \mathrm{~mm})$. O aquecimento do reator e do pré-aquecedor é realizado através de dois fornos bipartidos (5), equipados, cada um, com duas resistências infravermelhas em cerâmicas (Corel-Brazil) com 


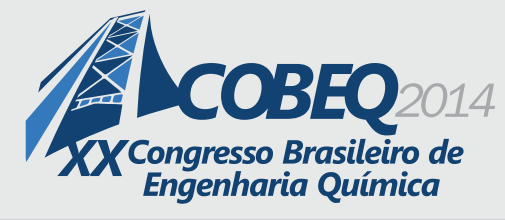

19 a 22 de outubro de 2014

Florianópolis/SC

potência de 1000 W. O acionamento das resistências é realizado por controladores (NovusBrazil), conectados aos termopares do tipo $\mathrm{J}$ (precisão $\left.\pm 1,0{ }^{\circ} \mathrm{C}\right)\left(\mathrm{T}_{1}\right)$ em contato ao pré-aquecedor e reator. Após percorrer o reator, a mistura resultante segue por um tubo de aço inoxidável 316 (L $=2,5 \mathrm{~m}$, I.D. $=3,175 \mathrm{~mm}$ ) para troca de calor com o ambiente externo, com monitoramento da temperatura realizado por um indicador de temperatura da (Novus-Brazil), conectado a um termopar tipo $\mathrm{T}$ (precisão $\left.\pm 1.0^{\circ} \mathrm{C}\right)\left(\mathrm{T}_{2}\right)$. Em seguida, a mistura resultante entra no condensador (7), uma serpentina de aço inoxidável $316(\mathrm{~L}=2,5 \mathrm{~m}, \mathrm{I} . \mathrm{D} .=3,175 \mathrm{~mm})$, para resfriamento à temperatura de $10{ }^{\circ} \mathrm{C}$. Após, a mistura resultante chega à válvula back-pressure $\left(\mathrm{V}_{2}\right)$, para ajuste da pressão, observado no manômetro (8). No coletor/separador de fases (9) ocorre a separação entre a fase líquida e vapor. A fase líquida é composta por água e lactose não reagida e outros produtos formados na reação e a fase vapor composta pelos produtos gasosos da reação, principalmente por, $\mathrm{H}_{2}, \mathrm{CO}, \mathrm{CO}_{2}$ e $\mathrm{CH}_{4}$.

\subsection{Análises}

A concentração de carbono orgânico total foi obtida pela diferença entre a concentração de carbono total e a de carbono inorgânico. As amostras foram filtradas em membrana $0,45 \mu \mathrm{m}$ e acidificadas até $\mathrm{pH} 2$ e armazenadas a $4{ }^{\circ} \mathrm{C}$, para posterior análise em um analisador de carbono, marca Shimadzu - Total Organic Carbon Analyzer - modelo TOC-L. A redução do COT será calculada de acordo com a equação 1 .

$$
\text { Redução_TOC }(\%)=\frac{T O C_{\text {entrada }}-T O C_{\text {saida }}}{T O C_{\text {entrada }}} \times 100
$$

\section{RESULTADOS E DISCUSSÃO}

\subsection{Reação não-catalítica}

A redução de TOC foi verificada observando os efeitos da pressão, concentração mássica, temperatura, vazão de alimentação, catalisadores e oxidantes. Inicialmente, foram realizadas medidas de TOC para as reações não catalíticas para verificação da influência dessas variáveis. A tendência de comportamento da redução do TOC é averiguada na Figura 2. Onde verifica-se que a redução do TOC é influenciada principalmente pela elevação da temperatura. Ou seja, na temperatura de $550{ }^{\circ} \mathrm{C}$ a redução do TOC é aproximadamente $50 \%$, enquanto na temperatura de $700{ }^{\circ} \mathrm{C}$, a redução fica próximo aos $95 \%$. 


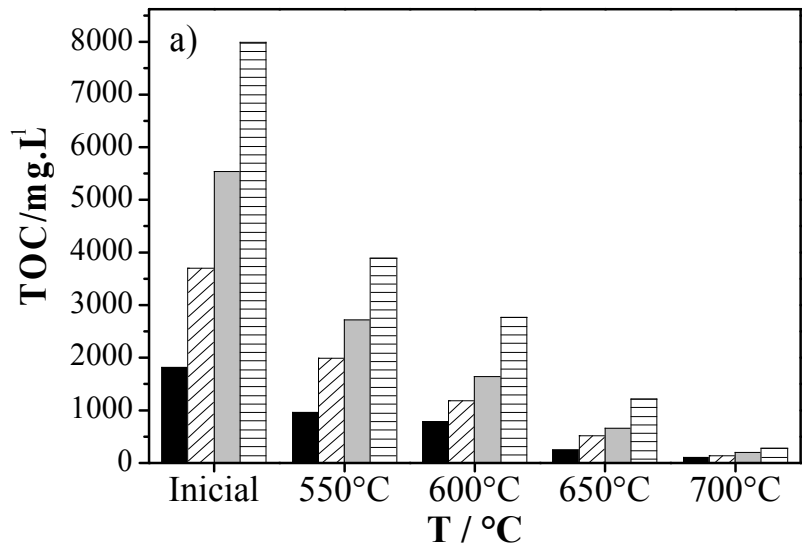

Concentração mássica / wt $\%$ : $1.5 \mathrm{wt} \%$ $2.5 \mathrm{wt} \%$

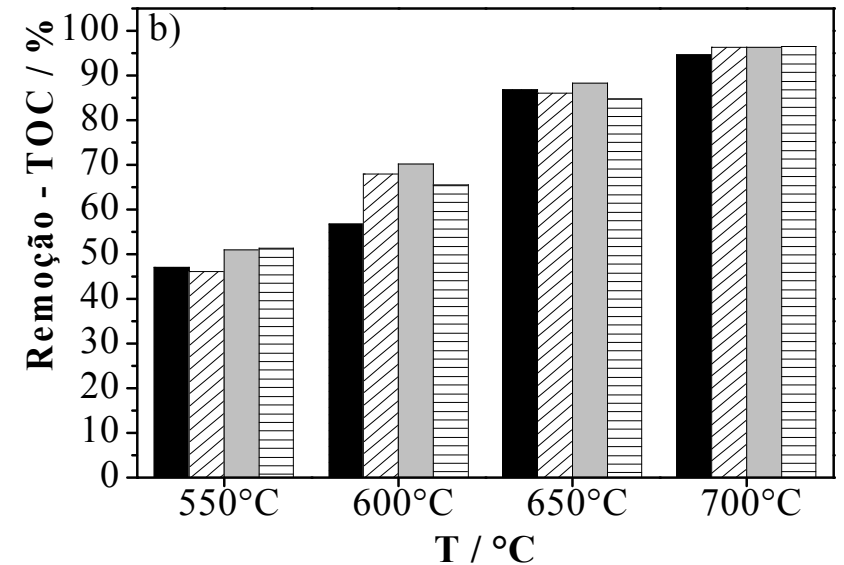

Concentração mássica/ wt $\%$ :
$1.5 \mathrm{wt} \%$ $2.5 \mathrm{wt} \%$

Figura 2 - Efeito da temperatura na remoção do TOC na ScWG da lactose a 22,5 MPa e 5 g.min ${ }^{-1}$ - (a) Valores iniciais e finais de TOC e (b) Percentual de remoção de TOC.

\subsection{Reação catalítica}

A adição de catalisadores reduziu significativamente o TOC das amostras. A maior contribuição na redução do TOC foi do $\mathrm{Na}_{2} \mathrm{CO}_{3}$, alcançando 97\%. O $\mathrm{Na}_{2} \mathrm{CO}_{3}$ reduziu significativamente o TOC em todas as massas de catalisadores estudados $(0,1 \mathrm{wt} \%, 0,3 \mathrm{wt} \%$ e 0,5 wt \%). O NaOH foi o menos eficaz na redução do TOC dentre os três catalisadores estudados, conforme Figura 3. A eficiência dos catalisadores na redução de TOC tem a sequência $\mathrm{Na}_{2} \mathrm{CO}_{3}>$ $\mathrm{KOH}>\mathrm{NaOH}$. 

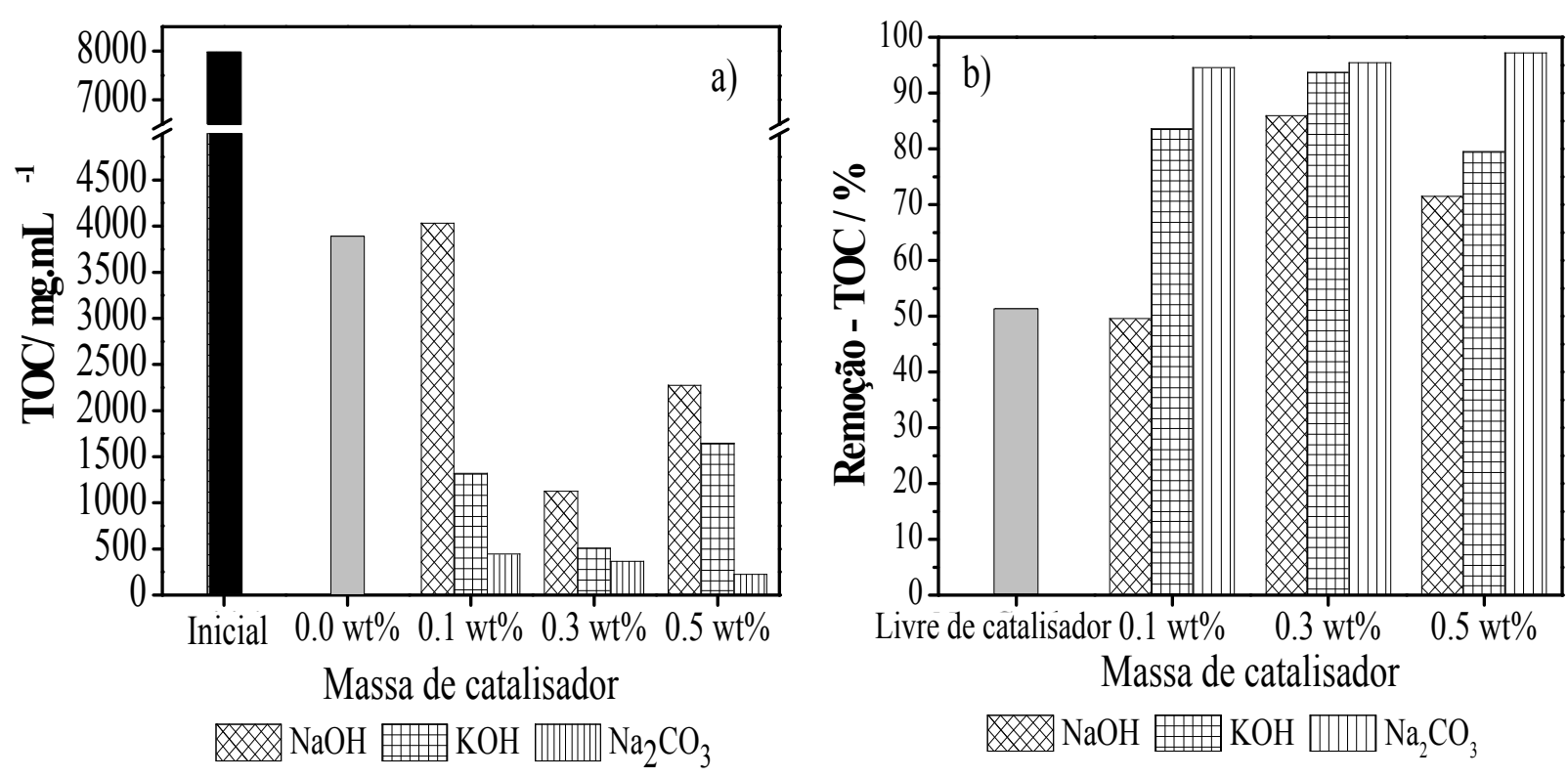

Figura 3 - Efeito da quantidade de diferentes catalisadores na remoção do TOC a partir da ScWG da lactose $22,5 \mathrm{MPa}, 550^{\circ} \mathrm{C}$ e 5 g. $\mathrm{min}^{-1}$ : (a) Valores iniciais e finais de TOC; (b) Percentual de remoção de TOC.

\subsection{Reação oxidativa}

A adição de oxidantes reduziu significativamente o TOC do efluente quando comparado ao efluente de entrada. As análises de TOC foram realizadas para as temperaturas de $550{ }^{\circ} \mathrm{C}$ e 700 ${ }^{\circ} \mathrm{C}$, obtendo reduções de até $99 \%$. Conforme esperado, a temperatura de $700{ }^{\circ} \mathrm{C}$ apresentou menores valores de TOC, principalmente nas concentrações $0,75 \mathrm{wt} \%$ e $1,5 \mathrm{wt} \%$, Figura 4 . Percebe-se que nas concentrações de $3,0 \mathrm{wt} \%$ e $5,0 \mathrm{wt} \%$ a temperatura não influenciou significativamente, pois, para ambas as temperaturas a redução do TOC foi semelhante. Contudo, o $\mathrm{H}_{2} \mathrm{O}_{2}$ apresentou maiores reduções de TOC quando comparados ao THB, principalmente na temperatura de $550{ }^{\circ} \mathrm{C}$. Nesta temperatura, o THB apresentou reduções de TOC muito inferiores às reduções obtidas com o $\mathrm{H}_{2} \mathrm{O}_{2}$. Contudo, para a temperatura de $700{ }^{\circ} \mathrm{C}$ essa diferença não foi observada. 


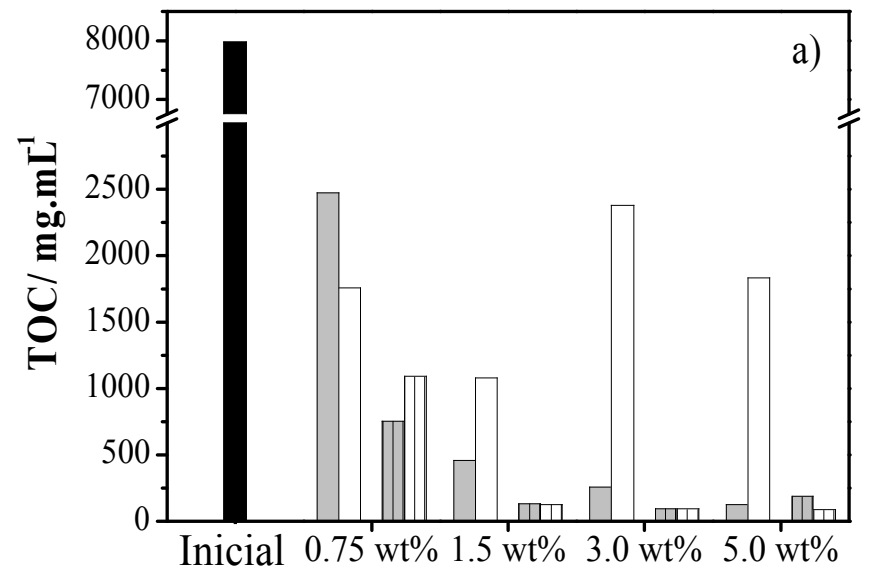

Massa de oxidante

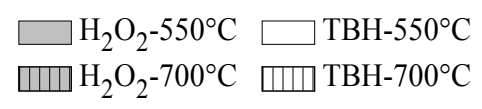

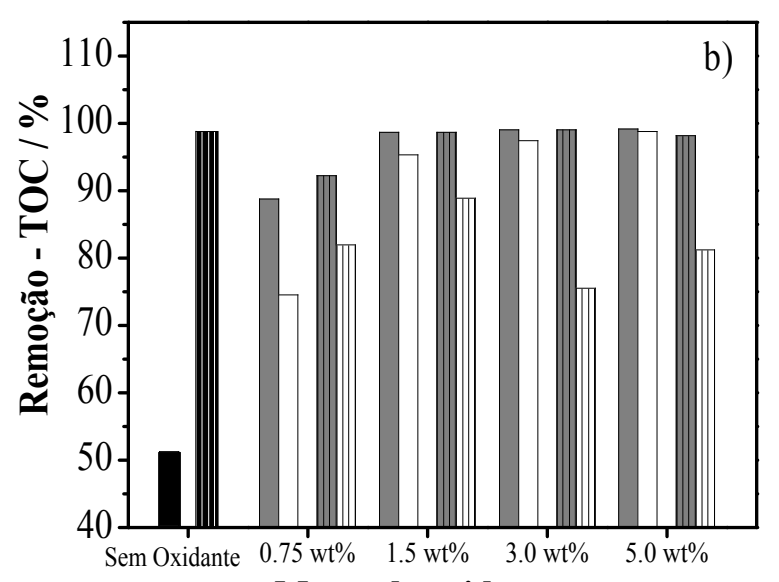

Massa de oxidante

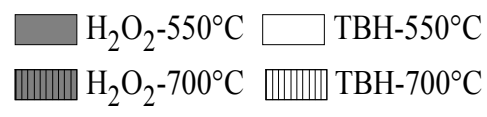

Figura 4 - Efeito da quantidade de diferentes catalisadores na remoção do TOC a partir da ScWG da lactose: (a) Valores iniciais e finais de TOC; (b) Percentual de remoção de TOC $\left[550{ }^{\circ} \mathrm{C}\right.$ (símbolo hachurado) e $700{ }^{\circ} \mathrm{C}$ (símbolo não-hachurado)].

\section{CONCLUSÃO}

Este trabalho teve como proposta estudar a oxidação da lactose em água supercrítica. Onde, verificou-se que a elevação da temperatura foi um fator primordial na redução eficiente do TOC, pois, em reações a $550^{\circ} \mathrm{C}$ a redução do TOC foi de $\sim 50 \%$, enquanto que, a $700{ }^{\circ} \mathrm{C}$ a redução foi de $\sim 96 \%$. No entanto, a utilização de sais alcalinos ou oxidantes em reações a $550{ }^{\circ} \mathrm{C}$ elevou de $\sim 50 \%$ para $97 \%$ a redução do TOC. Desta maneira, verifica-se que os dados obtidos neste trabalho podem servir como base inicial para futuros trabalhos que envolvam efluentes de laticínios. Pois, verificou-se que a metodologia de oxidação supercrítica da lactose apresentou resultados interessantes de redução de TOC, sendo uma alternativa considerável no tratamento de efluentes.

\section{AGRADECIMENTOS}

Agradecemos aos suportes financeiros concedidos pelo CNPq e Fundação Araucária/Paraná.

\section{REFERÊNCIAS}

ABELlEIRA, J., SÁNCHEZ-ONETO, J., PORTELA, J.R., MARTÍNEZ DE LA OSSA, E.J. Kinetics of supercritical water oxidation of isopropanol as an auxiliary fuel and co-fuel. Fuel 111, p. 574-583, 2013.

BERMEJO, M.., COCERO, M.., FERNÁNDEZ-POLANCO, F. A process for generating power from the oxidation of coal in supercritical water. Fuel 83, p. 195-204, 2004. 


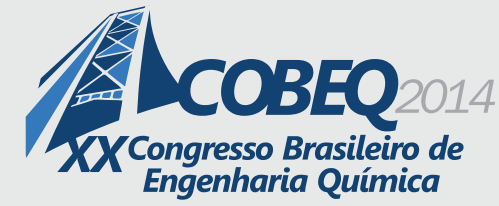

BRIÃO, V.B., TAVARES, C.R.G. Effluent generation by the dairy industry: preventive attitudes and opportunities. Brazilian J. Chem. Eng. 24, p. 487-497, 2007.

BRIÃO, V.B., TAVARES, C.R.G. Nota Científica: ultrafiltração de efluente da indústria de laticínios para recuperação de nutrientes: efeito da pressão e da velocidade tangencial. Brazilian J. Food Technol. 15, p. 352-362, 2012.

BRUNNER, G. Near and supercritical water. Part II: Oxidative processes. J. Supercrit. Fluids 47, p. 373-381, 2009.

COCERO, M.., ALONSO, E., SANZ, M.., FDZ-POLANCO, F. Supercritical water oxidation process under energetically self-sufficient operation. J. Supercrit. Fluids 24, p. 37-46, 2002.

DEMIREL, B., YENIGUN, O., ONAY, T.T. Anaerobic treatment of dairy wastewaters: a review. Process Biochem. 40, p. 2583-2595, 2005.

García Jarana, M.B., Sánchez-Oneto, J., Portela, J.R., Nebot Sanz, E., Martínez de la Ossa, E.J. Supercritical water gasification of industrial organic wastes. J. Supercrit. Fluids 46, p. 329-334, 2008. GLITHERO, N., CLARK, C., GORTON, L., SCHUHMANN, W., PASCO, N. At-line measurement of lactose in dairy-processing plants. Anal. Bioanal. Chem. 405, p. 3791-9, 2013.

HELLSTRÖM, D., BAKY, A., JEPPSSON, U., JÖNSSON, H., KÄRRMAN, E. Comparison of Environmental Effects and Resource Consumption for Different Wastewater and Organic 1Waste Management Systems in a New City Area in Sweden. Water Environ. Res. 80, p. 708-718, 2008.

JIMENEZ-ESPADAFOR, F., PORTELA, J.R., VADILLO, V., SÁNCHEZ-ONETO, J., BECERRA VILLANUEVA, J.A., TORRES GARCÍA, M., MARTÍNEZ DE LA OSSA, E.J. Supercritical Water Oxidation of Oily Wastes at Pilot Plant: Simulation for Energy Recovery. Ind. Eng. Chem. Res. 50, p. 775-784, 2011.

SARAIVA, C.B., MENDONÇA, R.C.S., SANTOS, A.L., PEREIRA, D.A. Water consumption and effluent generation in a small dairy industry. Rev. Inst. Laticínios Cândido Tostes 64, p. 10-18, 2009. SAVAGE, P.E. Organic Chemical Reactions in Supercritical Water. Chem. Rev. 99, 603-622., 1999. SILVA, A.C., DEZOTTI, M., SANT'ANNA, G.L. Treatment and detoxification of a sanitary landfill leachate. Chemosphere 55, p. 207-14, 2004.

SMITH, D.W., WANG, F. Application of advanced oxidation methods for landfill leachate treatment - a review. J. Environ. Eng. Sci. 2, p. 413-427, 2003.

SÖĞÜT, O.Ö., KIPÇAK, E., AKGÜN, M. Treatment of whey wastewater by supercritical water oxidation. Water Sci. Technol. 63, p. 908-916, 2011.

VERIANSYAH, B., KIM, J.-D. RETRACTED: Supercritical water oxidation for the destruction of toxic organic wastewaters: A review. J. Environ. Sci. 19, p. 513-522, 2007.

VOURCH, M., BALANNEC, B., CHAUFER, B., DORANGE, G. Nanofiltration and reverse osmosis of model process waters fromthe dairy industry to produce water for reuse. Desalination 172, p. 245-256, 2005.

VOURCH, M., BALANNEC, B., CHAUFER, B., DORANGE, G. Treatment of dairy industry wastewater by reverse osmosis for water reuse. Desalination 219, p. 190-202, 2008.

XU, D., WANG, S., TANG, X., GONG, Y., GUO, Y., WANG, Y., ZHANG, J. Design of the first pilot scale plant of China for supercritical water oxidation of sewage sludge. Chem. Eng. Res. Des. 90, p. 288-297, 2012. 